\title{
Strates
}

STRATES Matériaux pour la recherche en sciences sociales

$7 \mid 1993$

Témoins du monde : Bulgarie, identités chinoises, explorer l'île de France

\section{Planification urbaine et régionale : leçons et perspectives des expériences étrangères}

\section{Catherine Rhein}

\section{(2) OpenEdition \\ Journals}

Édition électronique

URL : http://journals.openedition.org/strates/1150

ISSN : $1777-5442$

Éditeur

Laboratoire Ladyss

Édition imprimée

Date de publication : 30 juin 1993

ISSN : 0768-8067

\section{Référence électronique}

Catherine Rhein, «Planification urbaine et régionale : leçons et perspectives des expériences étrangères », Strates [En ligne], 7 | 1993, mis en ligne le 20 décembre 2005, consulté le 07 septembre 2020. URL : http://journals.openedition.org/strates/1150

Ce document a été généré automatiquement le 7 septembre 2020

Tous droits réservés 


\title{
Planification urbaine et régionale : leçons et perspectives des expériences étrangères
}

\author{
Catherine Rhein
}

1 L'opération de valorisation de la recherche urbaine sur l'Ile-de-France, que nous avons menée, Martine Berger et moi à la demande du Plan Urbain et avec son soutien, s'est achevée il y a un $a n^{1}$. Le texte proposé ici correspond à une réflexion en cours, imparfaite et incomplète par bien des aspects, menée peu après l'achèvement du rapport. Une interrogation, qui reste largement implicite, sous-tend cette réflexion : jusqu'où devons-nous et pouvons-nous nous investir dans la démarche planificatrice - en tant que géographes - ? Avons-nous su - nous, géographes - prendre et préserver un degré de liberté suffisant (et nécessaire) à l'égard des pratiques de planification et de leurs enjeux socio-politiques? Ou restons-nous dans cet « apolitisme très politisé » dont J-P Gaudin a qualifié la posture de certains géographes dans les années 1960 ?

2 La préparation du nouveau Schéma Directeur d'Aménagement et d'Urbanisme (SDAU) de l'Ile-de-France a mis au jour une série de problèmes qui, pour être directement liés au processus de décentralisation, n'en sont pas moins des questions socio-politiques et heuristiques de fond. Pour le planificateur, la tâche est, aujourd'hui, plus complexe que jamais, puisqu'il travaille sous trois contraintes, celle de l'équité territoriale et sociale, celle du respect de l'environnement, enfin celle de l'efficacité économique, dans un contexte de décentralisation administrative inconnu en France jusqu'au début des années 1980 .

3 La planification urbaine et régionale est, en France, récente par rapport à celles mises en oeuvre dans les pays anglo-saxons, celles-ci remontant à 1909 en Grande-Bretagne, et au début des années 1910 aux États-Unis, pays à tradition communautaire, par opposition à la France, de tradition jacobine, centralisatrice et républicaine. Pourquoi cette émergence précoce de procédures de planification locale dans ces pays de droit anglo-saxon (common law), par opposition à son apparition tardive en France et dans les pays de droit romain? Fortement et structurellement décentralisés, plusieurs pays anglo-saxons ont bu cette coupe jusqu'à la lie: des expériences allemandes, 
britanniques et américaines, quelles leçons tirer pour l'Ile-de-France et jusqu'où peut aller le transfert de politiques et de modèles?

Dans le même temps, élus et entrepreneurs de la CEI découvrent, au fil de leur "révolution démocratique $»^{2}$, l'importance du «développement territorial». Pour B. Sazonov, il y aurait là moyen de réinventer de nouvelles formes de démocratie, par un travail de "repolitisation", par la reconstitution de "sujets politiques »3. Bien que le détour puisse nous paraître bien long, peut-être y a-t-il là aussi d'autres leçons à tirer, pour les citoyens de démocraties parlementaires (paraît-il) vieillissantes.

Ainsi, l'urbanisme, "praxis ", est aussi et surtout pratique ${ }^{4}$, résultant d'une interface complexe entre plusieurs milieux d'architectes, d'ingénieurs et d'urbanistes, celui de la gestion locale, enfin celui de la politique; n'est-il pas désormais bien loin des objectifs que lui assignaient les réformateurs sociaux du début du siècle?

6 Pour C. Chaline ${ }^{5}$, «quels types de relation peuvent-ils exister entre des politiques d'aménagement répondant aux tendances profondes de la dynamique urbaine contemporaine et des formes et modes d'utilisation de l'espace urbain hérités d'un autre âge et gérés présentement selon des logiques spécifiques à chaque organisme concerné ?» Les approches comparatives apportent à cette question une dimension importante, notamment sur les conditions politiques propres à chacun des pays et sur leurs incidences sur les politiques foncières.

1. État(s) et planification urbaine et régionale : quels moyens?

7 Si les outils de planification régionale et urbaine/locale diffèrent assez peu, dans leur forme, d'un pays (développé) à l'autre, c'est que les influences réciproques ont été importantes à l'échelle internationale, dès le début du siècle, même si leurs effets peuvent ne s'être manifestés que tardivement. Dès ses origines, le milieu des urbanistes et des planificateurs urbains s'est constitué sur une base internationale: cette «science " nouvelle s'inscrivait d'emblée hors du cadre des États-nations et de leurs structures politico-administratives, cherchant à en dépasser ou à en nier les spécificités juridiques et socio-politiques ${ }^{6}$. Ces influences ont été d'autant plus marquées que l'urbanisme et la planification urbaine et régionale se développaient aux confins des appareils d'État, tant dans les systèmes fortement centralisés que dans les systèmes juridiquement et politiquement décentralisés, comme celui de l'Allemagne ou les ÉtatsUnis.

8 Ainsi le Schéma Directeur d'Aménagement et d'Urbanisme français apparaît proche, tout au moins dans sa conception, en tant qu'outil de planification régionale et métropolitaine, des master plans anglo-saxons. Encore faut-il spécifier que ceux-ci sont bien antérieurs à ceux-là, puisque les SDAU ont été institués par la Loi d'Orientation Foncière de 1968, tandis que les master plans remontent au début du XX $\mathrm{XX}^{\mathrm{e}}$ siècle. Parmi les tout premiers, le Burnham Plan de Chicago date de $1909^{7}$, comme le premier plan de New-York.

9 C'est sans doute davantage le dispositif londonien, préparé par le Barlow Report (1937-1940) et proposé dans le Greater London Plan élaboré sous la direction de P. Abercrombie en $1944^{8}$ qui a inspiré l'équipe du District de Paris, dirigée par P. Delouvrier, vingt ans plus tard. Pour autant, le contexte politique dans lequel le plan Abercrombie a été élaboré et mis en oeuvre était-il bien différent de celui du premier SDAU de l'Ile-de-France. 
Quant aux Plans d'Occupation des Sols (POS), leurs équivalents sont, aux États-Unis, les Zoning Codes, dont le premier est institué, à New-York pour la première fois en 1916 et a alors pour fonction essentielle de réglementer la hauteur des immeubles ${ }^{9}$. Mais J.P. Gaudin indique que la notion de «zone » et les procédures de planification par zone sont plus anciennes, puisqu'elles ont été instituées par voie législative en 1875 dans l'Empire allemand $^{10}: c^{\prime}$ est fort vraisemblablement de ce "modèle » allemand que sont inspirées les procédures de zoning britannique et américaines.

De fait, la manière anglo-saxonne dont est conçu le zonage renvoie à celle dont s'opère l'urbanisation dans les pays anglo-saxons : un developer prend en charge la construction et la promotion des logements sur des parcelles relativement vastes, dont l'opération de lotissement lui incombe aussi. Ainsi, le tissu pavillonnaire issu du cycle d'urbanisation des années 1920-1930 est beaucoup plus homogène dans les banlieues de Londres que dans celles de Paris. Cette manière anglo-saxonne de penser et d'« effectuer " l'urbanisme est très évidemment sous-tendue par celle de penser le droit (de propriété) et par une division du travail architectural, de promotion et de construction, fondée sur des principes et des structures financières et économiques différents de ce qu'ils sont en France. La «zone " comme ensemble de parcelles constituant un îlot ou un groupe d'îlots est, avant même l'institution de la procédure du zoning, l'unité d'action du developer.

Rétrospectivement, il apparaît donc logique que le législateur ait constitué cette échelle comme la plus pertinente pour l'établissement des règles d'utilisation et d'occupation du sol. En France, cette même échelle a été retenue, alors même qu'elle ne s'inscrit ni dans les mêmes pratiques, ni selon les mêmes découpages territoriaux. En effet, en toute hypothèse, la fragmentation de la propriété foncière y est beaucoup plus marquée et les formes de ségrégation socio-spatiale y sont beaucoup plus complexes, parce que plus diverses.

Hiérarchie urbaine et équipements

13 A une autre échelle, le classement et la hiérarchisation des villes selon leurs niveaux d'équipements constitue une des procédures de la planification régionale allemande qui lui appartiennent en propre. C'est une procédure qui n'est, en réalité, pas inconnue dans l'aménagement du territoire français, puisque les politiques des métropoles d'équilibre ${ }^{11}$ et des villes moyennes en constituaient des fragments. Ces deux politiques, comme celle des places centrales, trouvent leurs fondements théoriques, sinon historiques, dans la notion de "pôle de croissance » et la théorie du "développement régional polarisé", in fine dans le concept de multiplicateur, tels qu'ils ont été développés notamment par J.-R. Boudeville, à partir des travaux de l'équipe de F.Perroux ${ }^{12}$, eux-mêmes partiellement inspirés de ceux de W. Isard, qui s'était quant à lui inspiré des travaux d'A. Lösch et de W. Christaller ${ }^{13}$.

14 Première singularité de cette généalogie, M-C Robic a montré les origines françaises de cette théorie ${ }^{14}$ que W. Christaller pourrait avoir seulement repris et développé. Le saint-simonien Jean Reynaud, polytechnicien, avait en effet esquissé, dans les années 1840-1850, une véritable géographie théorique, aux fondements socio-économiques du reste assez riches : il proposait notamment une « organisation théorique du territoire fondée sur une hiérarchie de trois à quatre niveaux de centres rayonnant sur des hexagones emboîtés $»^{15}$.

15 Seconde singularité : si les travaux de l'économiste W. Christaller commencent à être connus en géographie française à la fin des années 1960, il ne semble pas que les 
applications de ses travaux pour l'aménagement du territoire, en particulier la hiérarchisation stricte du réseau urbain, qui a été instituée pendant la période national-socialiste, soient connues en France ${ }^{16}$.

Selon M. Rössler, c'est au cours de la période national-socialiste que se développent en Allemagne aménagement du territoire (Raumordnung) et science régionale (Raumforschung), toutes deux reposant sur cette théorie des places centrales : celle-ci repose sur un principe d'équité territoriale selon lequel, où qu'ils habitent, tous les citoyens (allemands) doivent pouvoir accéder à des services de santé, d'éducation et d'administration de même qualité.

Ainsi I. Ernst indique qu'en Allemagne, la planification régionale continue de reposer sur cette théorie des places centrales qui implique la hiérarchisation complète du réseau urbain en places centrales, selon leur importance et la qualité de leurs équipements. L'espace allemand est ainsi modelé de manière " rationnelle » : le modèle des places centrales en constitue le fondement théorique, en économie régionale ${ }^{17}$.

Les pratiques de planification en sont marquées, au point que les agglomérations urbaines font l'objet d'un classement en trois grandes catégories, selon qu'il s'agit de places centrales de rang supérieur, moyen ou inférieur. Les documents d'urbanisme proposent en outre des axes de développement, des «zones centrales» ainsi que les " marges de zones centrales", autant de concepts issus directement de la formalisation du réseau urbain telle que le proposait $W$. Christaller.

19 I. Ernst précise cependant qu'actuellement, les communes allemandes sont fréquemment en conflit avec les Länder sur ces procédures de classement, parce qu'elles souhaiteraient un assouplissement, voire une suppression pure et simple, de ces prescriptions, c'est-à-dire du classement des bourgs et villes en places centrales hiérarchisées.

20 Ce souci d'équité tend en effet actuellement à s'effacer devant les préoccupations environnementales. Ainsi plus de 150 villes ont réalisé des cartographies de biotopes et tendent à revendiquer une gestion territoriale très fine, à l'échelle de la parcelle et ces préoccupations environnementales entrent de plus en plus fréquemment en contradiction avec celles issues du principe de l'équité territoriale dans l'usage des sols. Aménagement public/privé

21 Autre procédure de planification - plus récente, plus ponctuelle -, la dévolution au secteur privé de l'aménagement d'un quartier est l'option pour laquelle a opté la Grande-Bretagne, en particulier dans le secteur des Docklands londoniens. Cependant, selon B. Goodchild ${ }^{18}$, le virage vers un tel modèle de " partenariat " privé/public s'était déjà largement amorcé à la fin des années 1970, alors que les municipalités travaillistes cherchaient tous les moyens de surmonter la double crise de leurs finances locales, pour fait de récession nationale et pour cause de désindustrialisation régionale. Les premières solutions ont donc été mises au point dans le cadre des programmes de réhabilitation/rénovation des inner cities. En particulier, le statut des Urban Development Corporations (UDC) avait été élaboré, instituant une nouvelle catégorie d'acteur, au statut juridique mixte et dont les moyens financiers sont essentiellement d'origine privée.

L'objectif principal des UDC a été d'inciter les investisseurs privés à intervenir sur des zones urbaines sur lesquelles les droits du sol étaient gérés, de manière dérogatoire, par l'UDC ${ }^{19}$. Le succès très relatif des UDC sera discuté plus loin. Pour B. Goodchild, les 
limites de telles associations entre secteurs privé et public dans le domaine de l'aménagement urbain sont nombreuses.

Tout d'abord, de telles interventions ne couvrent pas l'ensemble des équipements urbains : ainsi les transports en commun et le réseau routier continuent d'être à la charge de l'État ou d'organismes spécifiques, qui ne peuvent être partie prenante dans les UDC. De plus, les UDC encourent des risques importants de conflit entre leurs fonctions régulatrices et planificatrices et leur rôle de promotion du développement local.

Autre source de problèmes: les contraintes du marché font que les projets liés à la consommation privée ou collective sont beaucoup plus fréquemment retenus par les UDC que ceux liés au développement d'activités économiques et, en particulier, industrielles.

Pour les mêmes raisons et suivant la même logique, les projets localisés dans des sites privilégiés, notamment dans les centres anciens, sont plus fréquemment retenus que les autres. Là encore, les objectifs de redéveloppement ne sont donc pas véritablement atteints, puisqu'il est vraisemblable que ces sites auraient été redéveloppés de toute façon, sans intervention. En particulier, les municipalités, se faisant ainsi une concurrence acharnée pour attirer les investisseurs, améliorent ou créent des zones d'activités industrielles et commerciales: ainsi, tout comme dans certains secteurs d'Ile-de-France, l'offre de terrains industriels et de mètres carrés de bureaux a-t-elle finalement été pléthorique.

Enfin, cette procédure renforce le pouvoir des propriétaires fonciers, au détriment des considérations d'équité territoriale et des résidents de ces quartiers. Le bilan de cette forme d'aménagement est donc d'autant plus négatif que les investisseurs n'y trouvent pas nécessairement leur compte, comme l'a montré, tout récemment, la conclusion financièrement désastreuse de l'opération Canary Wharf.

2. Quelles structures et quelles échelles de gestion et de planification territoriale?

Ces systèmes de planification régionale diffèrent dans leurs modalités de fonctionnement, précisément parce qu'ils s'articulent sur des appareils de gestion territoriale aux structures variées et qu'ils se déploient dans des contextes sociopolitiques et dans des configurations politiques très différents.

La première question porte sur les rapports entre fédéralisme et planification urbaine et régionale. Fédéralisme et décentralisation de la planification sont-ils indissolublement liés? La relation apparaît, à l'examen, beaucoup moins simple. En effet, plusieurs travaux le montrent, l'impact du fédéralisme sur ces procédures et sur les techniques de planification varie selon l'échelle d'intervention.

29 Ainsi, dans la planification urbaine allemande, plus précisément encore dans la gestion de l'occupation du sol, c'est-à-dire à grande échelle, L. Hoffmann et A. Heymann-Doat concluent que "le fédéralisme est sans grande conséquence " ${ }^{20}$.

30 En revanche, à plus petite échelle, d'un Land à l'autre, les procédures de planification régionale ne sont pas identiques, bien que le classement des villes en places centrales en constitue le dénominateur commun. Parce que les Länder sont des États à part entière, les formes d'administration territoriale sont très différentes d'un Land à l'autre, mais aussi la taille des communes. Il en va de même pour les unités de planification, différentes aussi selon les Länder, puisque la planification régionale relève de la compétence législative des Länder. 
31 A une échelle intermédiaire, la centralisation des compétences est donc forte - de fait, sinon de droit -, puisque les Länder sont de taille relativement réduite. Aussi l'unité administrative, le Kreis, intermédiaire entre Land et commune, est-elle nécessairement petite : elle coïncide, en superficie, à la moitié d'un arrondissement français, alors qu'elle correspond au département dans le dispositif juridico-administratif.

32 Enfin, à un quatrième niveau, les communes jouissent, en principe, d'une autonomie relativement large, supérieure à celle des Kreise : la délivrance du permis de construire est ainsi confiée aux communes, comme en France.

33 Pour I. Ernst, les divisions politico-administratives ne constituent cependant pas un obstacle au développement économique et urbain. Au contraire, le réseau urbain polycentrique constitue un atout important, difficile à "penser » pour des Français trop rompus aux modèles fortement polarisés et en particulier au modèle de la métropole parisienne.

34 Le cas des États-Unis est très différent. S'il s'agit aussi de structures fédérales, les différences tiennent au degré d'autonomie beaucoup plus grand des collectivités territoriales aux États-Unis, et à la grande variété de leurs formes, par rapport aux Länder et aux communes allemandes.

Cette autonomie des collectivités territoriales américaines s'affirme jusque dans le processus proprement dit de leur constitution, notamment par cette procédure de l'incorporation, qui doit émaner d'une "communauté ", ou plus précisément d'un ensemble de résidents soucieux de se constituer en communauté, afin de gérer euxmêmes le territoire sur lequel ils résident. Cette opération politico-juridique peut être lue comme processus démocratique, exemplaire sous bien des rapports. En réalité, il est, à l'heure actuelle, utilisé comme processus de préservation d'un « entre-soi » socioethnique, comme l'indique C. Ghorra-Gobin ${ }^{21}$.

36 Si les formes d'organisation territoriale ont indéniablement un impact sur les modalités de planification spatiale, cet impact ne se situe pas exactement là où on l'attendrait. En particulier, les appareils de gestion territoriale fortement décentralisés n'engendrent pas nécessairement les formes de planification les plus décentralisées.

37 L'exemple le plus actuel et le plus topique est, de ce point de vue, celui de la région métropolitaine de Londres, par rapport à celle de l'Ile-de-France. En effet, depuis le début des années 1980, le gouvernement conservateur a déstabilisé, puis fait disparaître, en 1986, le Greater London Council, à majorité travailliste, structure relativement jeune pourtant dans l'histoire administrative de la région puisqu'il avait été institué en 1964., prenant - il est vrai - le relais d'une instance aux compétences plus réduites, le London Boroughs Council, créée à la fin du XIX ${ }^{\mathrm{e}}$ siècle.

38 L'une des conséquences les plus connues et les plus médiatisées de ce renversement politique et de cette "recentralisation» de facto a été l'aménagement des Docks londoniens. L'opération a été menée de manière relativement discrétionnaire et les collectivités locales n'ont guère été consultées. Plus encore, les Docklands, place forte de la classe ouvrière londonienne, devaient faire place à l'extension de la City vers l'Est. Mais l'échec financier d'une grande partie de l'opération Canary Wharf clôt sur une note amère, pour les néo-libéraux, un dossier politiquement lourd: le marché n'aura donc pas eu l'efficacité qu'ils lui prêtaient.

39 L'échec cuisant de l'opération de redéveloppement de Canary Wharf est lourdement symbolique, en cette fin de siècle, marquée par d'autres échecs idéologiques et 
politiques. Il indique les limites du recours au marché en matière d'aménagement urbain, mais aussi, a contrario, l'importance du rôle régulateur de l'État : la politique des grands travaux, très décriée, a permis de mener à bien des projets qui n'eussent sans doute pas pu aboutir par les procédures normales, décentralisées, d'aménagement urbain.

En Ile-de-France, la situation est, politiquement, l'opposée de celle de Londres : un gouvernement socialiste affronte une majorité régionale et un conseil municipal de Paris relevant tous deux de l'opposition. Sur certains dossiers, parisiens en particulier, le recours à la formule des "grands travaux" s'est avéré nécessaire pour faire progresser de manière (politiquement) significative des projets urbains de grande envergure.

41 La révision du Schéma Directeur pose en revanche de redoutables problèmes, dans la mesure où elle doit désormais reposer sur des procédures de consultation entre les différents niveaux de gestion (région, départements, communes). Par ailleurs, $l^{\prime}$ «ardente obligation » de la solidarité, qu'elle passe par des mesures fiscales ou par le développement de l'intercommunalité ${ }^{22}$ et le respect du schéma font d'autant plus problème que ce sont désormais les communes qui font et défont les POS et délivrent les permis de construire.

42 C'est essentiellement à l'échelle locale que les procédures d'élaboration des plans sont, le plus souvent, décentralisées, soit que la responsabilité en soit confiée aux instances élues locales, ou plus encore que cette élaboration fasse l'objet de véritables consultations des résidents.

La seconde formule a surtout été mise en œuvre aux États-Unis et au Canada, où elle est désignée sous le terme d'advocacy planning. Elle ne peut être mise en oeuvre qu'à l'échelle locale, celle d'une commune ou d'un quartier et présente le risque de ne pas être très prospective.

En poussant l'analogie un peu plus loin, il paraît possible de lire, dans les expériences de «jeux urbains" exposées par B. Sazonov ${ }^{23}$, une méthode d'advocacy planning qui transcende son objectif pour devenir une véritable maïeutique de la démocratie. Les acteurs en sont cependant différents; ils incluent les entrepreneurs et font aux entreprises naissantes une place qu'elles n'ont pas dans l'advocacy planning, forme actualisée et contrôlée des grass roots politics ${ }^{24}$.

45 La phase d'application des plans d'urbanisme est celle au cours de laquelle se posent, d'une part, le problème de la cohérence des différentes politiques publiques et, en second lieu, celui du respect des orientations définies dans les plans. In fine, c'est fréquemment sur la question de la crise du droit de l'urbanisme, quelle qu'en soit la forme, que débouchent les bilans de cette phase d'application des plans. A l'extrême, ce droit n'est tout simplement pas ou plus respecté, comme tel est le cas, notamment, dans les pays en voie de développement - qu'il s'agisse du Mexique ${ }^{25}$, du Brésil ${ }^{26}$, de $l^{1} I_{n d e}{ }^{27}$. Ou encore ce droit a induit des effets pervers tels qu'il est devenu nécessaire d'élaborer rapidement des parades, qu'il s'agisse les conséquences de la loi sur les logements des centres-villes, votée en Allemagne en 1972 ou des dérives des procédures de zoning, évoquées plus haut ${ }^{28}$.

3. Quels objectifs pour la planification spatiale?

46 C'est l'efficacité même de la planification spatiale qui semble actuellement remise en question. L'expérience des trente dernières années indique que ses effets sont, en 
réalité, plus limités que ce qui en était attendu et l'émergence de nouvelles contraintes et de nouveaux objectifs rend d'autant plus complexe son élaboration et sa mise en œuvre.

L'efficacité économique

Dans quelle mesure l'efficacité économique peut-elle être considérée comme un objectif pour la planification spatiale? Dans son principe, la planification a principalement pour objectif d'assurer une meilleure cohérence des projets urbains privés et publics et une relative socialisation des bénéfices de la croissance urbaine: aussi cet objectif d'efficacité économique est-il souvent second ou médiat, derrière celui d'équité territoriale, même s'il est loin d'être absent ${ }^{29}$.

Toute la question est de savoir de quelle efficacité économique il s'agit. La planification spatiale étant, en principe, une affaire d'État, ce n'est effectivement pas la rentabilité à court terme des entreprises privées qui est a priori visée, mais une efficacité médiate, à plus long terme, fondée sur un meilleur développement des forces productives, dont l'État doit se considérer comme garant. Or actuellement, la multiplication des intervenants dans le processus de planification et les responsabilités financières accrues auxquelles ces intervenants sont astreints font voler en éclats cette unité (apparente) et cette cohérence (présumée) de l'État et introduisent en son sein des impératifs de gestion inconnus jusqu'alors.

Enfin, l'inefficacité de la planification tient aussi, pour B. Goodchild ${ }^{30}$, J. Busquets ${ }^{31}$ et JP. Lacaze ${ }^{32}$, à sa trop grande dépendance à l'égard de la gestion territoriale et à une surévaluation de l'efficacité de ses outils. Ainsi des POS, dont J-P. Lacaze rappelle que « jusqu'à la date charnière de 1975, on a fait de l'urbanisme pour savoir où et comment construire. Depuis lors, dans le plus grand nombre des cas, c'est l'observation concrète des marchés fonciers et des modes d'ajustement de l'offre et de la demande en matière de logement qui permet de décider où et pourquoi il est nécessaire de faire de l'urbanisme. » Les changements de POS sont beaucoup plus rapides que les effets qu'ils sont censés produire à moyen et plutôt à long termes. Ainsi les POS sont-ils actualisés tous les dix ans, voire plus fréquemment, tandis qu'une simple mise à l'alignement d'une rue peut prendre un siècle : c'est en ce sens qu'il est possible de conclure à une relative dépendance à l'égard du marché. Dans quelle mesure cet instrument de planification peut-il alors jouer un rôle régulateur?

Équité territoriale et justice sociale

50 Par nature et dès ses origines, l'urbanisme est prescriptif et normatif. Il est plus particulièrement fondé sur une notion, sinon un concept, $d^{\prime}$ ' ordre » urbain et social, implicite dans les procédures fonctionnalistes de zoning. L'amélioration des conditions de vie et, tout particulièrement, des conditions de logement devait amener celle des modes de vie et des comportements de la classe ouvrière, pour les réformateurs sociaux.

51 Pour B. Goodchild ${ }^{33}$, ce bilan de la planification en matière de justice sociale est mince, à l'heure actuelle. En d'autres termes, la planification n'a eu, sur la " désorganisation " et le « désordre » supposés des grandes villes et des couches ouvrières qui les habitent, qu'un effet cosmétique : faut-il s'en réjouir ou le déplorer?

52 Il apparaît même que les procédures de zoning aient constitué, aux États-Unis, un puissant facteur de fragmentation et de ségrégation sociales. Ces processus de 
ségrégation sociale ou socio-ethnique se sont profondément accentués au cours de la dernière décennie reaganienne, dans certaines grandes métropoles américaines.

Certes les formes de ségrégation résidentielle ne sont jamais ni uniquement ethniques ni exclusivement sociales et leur lecture ne doit pas se limiter à leur aspect strictement résidentiel. Les exclus du marché du travail sont aussi concentrés dans des ghettos résidentiels, vastes zones urbaines dont les logements sont totalement décrépits, les équipements publics laissés à l'abandon et dont les autorités civiles se sont retirées. L'extension actuelle des ghettos noirs des grandes métropoles américaines ne peut donc être considérée seulement comme un échec de la planification urbaine américaine: elle a d'autres dimensions, macro-économiques et politiques, qui ne peuvent être développées ici.

54 La planification urbaine américaine n'est pas fondée sur un principe de solidarité entre collectivités territoriales d'une même agglomération. Elle est surtout efficace dans ce qui constitue sa mission principale, c'est-à-dire le contrôle de l'usage des sols et le respect des règlements d'urbanisme : elle sert davantage à protéger les collectivités les plus aisées de tout risque de "mixité sociale " qu'à oeuvrer pour une plus grande équité territoriale, comme le montrent C. Ghorra-Gobin ${ }^{34}$ et V. Renard ${ }^{35}$ et les moyens juridiques mis au point pour atténuer, sinon éliminer, cette dérive n'ont eu jusqu'alors qu'une efficacité limitée, dans un contexte globalement néo-libéral.

L'efficacité du zonage intégré (inclusionary zoning) semble effectivement limitée, pour V. Renard ${ }^{36}$. L'affaire remonte à 1962 : l'un des juges de la Cour Suprême du New Jersey critique le POS d'une ville, estimant que "la notion d'intérêt général transcende les limites artificielles des territoires des municipalités et ne peut se limiter à des politiques locales trop étroites ». Cette municipalité refusait, par le biais des règles d'urbanisme, l'accès de son territoire à des populations relativement modestes.

La démarche du juge Hall n'aboutit qu'en 1975 : la décision Mount Laurel ouvre une nouvelle ère, donnant raison à la National Association for the Advancement of Coloured People (NAACP) contre la municipalité de Mount Laurel (New Jersey) : cette dernière avait en effet élaboré un plan d'urbanisme socialement exclusif, plan dont le juge Hall obtient l'annulation, à la requête de la NAACP. Pour rendre effectif ce principe, et confirmer la jurisprudence Mount Laurel, la Cour Suprême du New Jersey énonce, en janvier 1983, dix principes généraux dont l'un est constitué par l'obligation, pour les municipalités, d'élaborer des plans locaux de l'habitat et de garantir une proportion minimale de logements accessibles aux ménages modestes dans les programmes de construction neuve.

Plusieurs États, notamment la Californie, ont dès lors modifié leur législation sur l'urbanisme et le logement de telle sorte que les municipalités soient contraintes de procéder à un zonage intégré. Mais les obstacles sont nombreux: le zonage intégré réduit la rentabilité des opérations immobilières, il implique aussi la mise en place d'un dispositif de contrôle des loyers et du prix des logements, celui de mesures d'incitation à inclure des logements peu coûteux dans les opérations et de critères d'attribution des logements.

En France, avant la décentralisation, l'excessive concentration et la trop grande centralisation des pouvoirs ont été constamment dénoncées, sans que l'on s'interroge alors sur les effets pervers ou peu souhaitables de la décentralisation. En la matière, l'expérience des pays fortement décentralisés - Allemagne et Etats-Unis - indique que 
certains risques existent et qu'ils ont pour nom fragmentation, voire ségrégation: l'impératif de solidarité n'en est que plus difficile à assurer.

Démocratie locale et risques de fragmentation politique

En Allemagne, selon I. Ernst, « le juridique a une place importante dans l'aménagement, alors qu'en France on pratique l'urbanisme de la circulaire $»^{37}$. La place du droit dans la vie administrative allemande est omniprésente. S'il n'existe pas d'équivalent allemand à l'École Nationale d'Administration, l'écrasante majorité des hauts fonctionnaires sont des juristes de formation et le rôle des tribunaux, important, explique notamment les nombreux recours des écologistes. L'Allemagne peut être vue comme une "société dissensuelle ", où le recours en contentieux est très fréquent.

Les débats sont nombreux pour l'élaboration des documents de planification: le «principe de contre-courant» veut que "pour toute disposition imposée par une instance supérieure, il y ait consultation des personnes ou institutions situées à un niveau territorial inférieur ». Dans les faits, la "décentralisation» est relative : si l'ouverture des débats, la dimension démocratique de ces pratiques sont indéniables, leur contrepartie est la lourdeur et la lenteur de la mise au point des documents de planification. C'est ce qui explique le recours trop fréquent à des pratiques dérogatoires et à des négociations serrées pour éviter tout recours juridique.

61 Pour I. Ernst, la crise de la planification allemande a pour origine un conflit opposant communes et Länder, les communes étant pour un assouplissement, voire une suppression pure et simple des prescriptions d'urbanisme, en particulier ce classement des places centrales évoqué plus haut, tandis qu'un certain regain d'intérêt pour la planification se fait jour dans les nouveaux Länder de l'ex-RDA, sans qu'il y ait, pour autant, renouvellement ou actualisation des procédures de planification.

Depuis plus d'un siècle, la pression démographique s'exerce suffisamment fortement pour que l'appareil de planification ait été développé de façon précoce et qu'il soit actuellement omniprésent dans l'appareil administratif. Réciproquement, la pression sur l'espace n'a cessé de s'accroître: l'afflux de réfugiés, puis les impératifs de redéploiement économique ont laissé, sur les paysages et les écosystèmes, des traces profondes, difficiles à gérer pour les planificateurs.

Autre symptôme, les grass roots politics américaines n'ont pas reçu, en France, toute l'attention qu'elles méritent. Niveau fondamental de l'émergence du fait politique, à l'échelle locale, il est seulement en cours de constitution en France depuis la décentralisation, à propos des POS, mais la fiscalité locale n'en constitue pas encore un enjeu.

En matière de POS, les positions de J-P. Lacaze sur l'urbanisme participatif ${ }^{38}$ restent très en deçà de la réalité. La question n'est plus de prendre en compte « l'espace tel qu'il est perçu et vécu par les habitants ${ }^{39}$. Il est en effet temps de dépasser l'angélisme condescendant dans lequel la plupart des élus et des experts se drapent et de constater qu'à l'instar des mouvements américains (grass roots politiques), la mobilisation locale se fait désormais, en France, plutôt dans les communes aisées, autour d'enjeux relativement égoïstes, tels que la préservation d'espaces verts privés et de faibles densités: la défense de l'environnement sert le plus souvent de prétexte à la préservation de positions acquises plus qu'à celle d'un « environnement » qui n'a plus grand-chose de naturel. Il faut aussi indiquer que ce type de mouvement a d'autant plus 
de chances d'aboutir que les enjeux ne sont pas d'intérêt général ou, à tout le moins, régional.

Ainsi, le bouclage de l'autoroute A14 en Ile-de-France comme celui de l'A86 vont finalement aboutir, en dépit d'une forte mobilisation de personnalités politiques et d'experts résidant dans les sites affectés par le passage de ces infrastructures de transport. En revanche, la modification d'un POS favorisant la relative densification d'une zone pavillonnaire située à proximité d'une station RER est aisément annulée, parce que les trente ménages concernés et "menacés » ont su protester et ont été entendus et que les bénéfices électoraux priment en ce cas sur l'intérêt général.

Conclusions

Le dilemme marché/Plan n'est pas dépassé, il se déplace: le jeu du marché est, aujourd'hui comme hier, insuffisant à assurer une extension et un développement satisfaisants des métropoles. En la matière, l'échec d'une des plus grosses opérations des Docklands londoniens est patent. La nouveauté tient dans l'interpénétration croissante des secteurs public et privé.

La combinaison entre plan et marché apparaît, en définitive, la solution la plus fréquente et la moins mauvaise : elle reste à inventer dans les pays ex-socialistes, où il est très vraisemblable qu'un retour à une planification de type occidental est fortement prévisible. L'expérience dont fait état B. Sazonov donne à penser que Moscou est plutôt, à l'heure actuelle, un véritable laboratoire dont pourraient sortir des figures tout à fait inédites de planification: la planification y est ici mère, et non plus fille, d'une démocratie aux contours encore bien incertains.

\section{NOTES}

1. Martine BERGER et Catherine RHEIN, 1992, L'Ile-de-France et la recherche urbaine, Paris, Plan Urbain-CNRS, 2 vol. 187 p. et 210 p.

2. J. BIDET et al., 1990, Fin du communisme, renouveau du marxisme ? Paris, PUF, p. 6.

3. B. SAZONOV, « Nouveaux concepts pour la gestion urbaine à Moscou, le jeu de l'action territoriale ", Annales de la Recherche Urbaine, n 51, 1992.

4. J-P. LACAZE, 1990, Les méthodes de l'urbanisme, Paris, PUF, $\mathrm{p} 7$.

5. C. CHALINE, «Éléments pour un débat franco-britannique », in Les grands propriétaires fonciers urbains, Paris, ADEF, 1987, pp. 7-12.

6. La constitution et les modalités de fonctionnement de ce milieu des urbanistes sont analysées par J-P. GAUDIN dans le chapitre II de L'aménagement de la société, Paris, Anthropos, 1979, et dans les chapitres II.1. et II.2 de L'avenir en plan, Champ Vallon, 1985. Ils sont aussi abordés par T. LEROUX dans « H. Sellier, maître d'oeuvre de la vie urbaine » (pp. 83-95) et par A. SUTCLIFFE dans « Le contexte urbanistique de l'oeuvre d'Henri Sellier : la transcription du modèle anglais de la cité-jardin » (pp. 67-79) in K.BURLEN (dir.), 1987, La banlieue-oasis, Henri Sellier et les cités-jardins (1900-1940), Vincennes, Presses Universitaires de Vincennes. 
7. H. M. MAYER and R. C. WADE, 1969, Chicago, growth of a metropolis, Chicago, Univ. of Chicago Press, pp. 274-280.

8. C. CHALINE, 1967, La métropole londonienne, Paris, A.Colin; P. MERLIN et P. GUERTIN, 1967, L'urbanisme dans la région de Londres et l'aménagement du territoire, Paris, rapport à l'IAURP.

9. J. HEFFER, 1972, New York, La Documentation française, p 24 ; C. CARDIA, 1985, New York à travers le XIX ${ }^{e}$ siècle, Paris, Thèse de 3ème cycle, EHESS, pp. 326-338.

10. J-P GAUDIN, L'avenir en plan, op. cit., pp. 63 et 110.

11. M. ROCHEFORT, C. BIDAULT et M. PETIT, 1970, Aménager le territoire, Paris, Paris, Le Seuil, p. 49 et suiv.

12. J-R. BOUDEVILLE (dir.), 1968, L'espace et les pôles de croissance, Paris, Presses Universitaires de France ; F. PERROUX, L'économie du XXème siècle, Paris, Presses Universitaires de France, note 1, p. 165 (éd. de 1969).

13. W. CHRISTALLER, 1966, Die zentralen Orte in Süddeutschland, Thèse de doctorat d'économie, Jena, 1933 ; Central Places in Southern Germany, Prentice Hall.

14. M-C. ROBIC, 1982, "Cent ans avant Christaller : une théorie des lieux centraux », L'Espace Géographique, vol.XI, pp. 5-12.

15. M-C. ROBIC, 1982, art. cit., p. 7.

16. M. RÖSSLER, 1988, « Géographie et national-socialisme ", L'Espace Géographique, vol.XVII, $n^{\circ} 1,5-14$, et 1989, « Applied geography and area research in Nazi society : central place theory and planning », Environment and Planning D, vol.7, 419-431.

17. I. ERNST, « La planification spatiale allemande, contexte et valeurs », Annales de la Recherche Urbaine, $\mathrm{n}^{\circ}$ 51, 1992, pp. 83-85.

18. B. GOODCHILD, « La planification post-moderne en Grande-Bretagne », Annales de la Recherche Urbaine, ${ }^{\circ} 51,1992$, pp. 112-121.

19. L'UDC a pour équivalent français l'Établissement Public d'Aménagement (EPA), à ceci près que les EPA interviennent principalement en villes nouvelles et à la Défense, secteurs urbains dont les caractéristiques sont opposées à celles dans lesquelles interviennent les UDC britanniques.

20. L. HOFFMANN et A. HEYMANN-DOAT, «L'évolution de la planification locale en République Fédérale Allemande », in A. HEYMANN-DOAT (coord.), 1983, Politiques urbaines comparées, Paris, A. Colin, p. 130.

21. C. GHORRA-GOBIN, «Planification urbaine et incorporation aux Etats-Unis, les fondements de la politique d'exclusion », Annales de la Recherche Urbaine, n 51, 1992. 22. Telle qu'elle est instituée par la loi du 13 mai 1991 sur le rééquilibrage de la Dotation globale de fonctionnement, sur l'instauration d'un mécanisme de péréquation propre à l'Ile-de-France et sur l'incitation à une plus grande solidarité intercommunale. 23. B. SAZONOV, « Nouveaux concepts pour la gestion urbaine à Moscou, le jeu de l'action territoriale » Annales de la Recherche Urbaine, n 51, 1992.

24. Ces grass roots politics, peu connus en France, sont la forme élémentaire de la démocratie américaine : de nombreux travaux de sociologie politique leur ont été consacrés, de SELZNICK - qui a traité en particulier de leurs dérives possibles - à M. CASTELLS.

25. E. DUHAU, 1992, «La régularisation de l'habitat au Mexique », Annales de la Recherche Urbaine, $\mathrm{n}^{\circ} 51$, p. 50 et suiv.

26. B. SOUTO-MAIOR FONTES, 1992, «Le discours des plans d'urbanisme à Recife ", Annales de la Recherche Urbaine, $\mathrm{n}^{\circ} 51$, p. 57 et suiv. 
27. I. MILBERT, 1992, « Réglementer la croissance ou définir le développement des villes indiennes? » Annales de la Recherche Urbaine, $\mathrm{n}^{\circ}$ 51, p. 63.

28. Sur l'Allemagne, L. HOFFMANN et A. HEYMANN-DOAT, op.cit. ; sur les États-Unis, V. RENARD, art.cit., C. GHORRA-GOBIN, art.cit.

29. Sur ce point, J-P. GAUDIN précise la genèse et les liens entre planification économique et planification régionale en France in L'Aménagement de la société, chap.III, 1979.

30. B. GOODCHILD, « La planification urbaine post-moderne en Grande-Bretagne », Annales de la Recherche Urbaine, $\mathrm{n}^{\circ}$ 51, 1992, p. 113.

31. J. BUSQUETS, « La planification-cadre et les projets-actions, l'exemple de Barcelone ", Annales de la Recherche Urbaine, n 51, 1992, p. 129.

32. J-P LACAZE, Méthodes de l'urbanisme, op.cit, p. 80.

33. Art.cit., p. 113.

34. C. GHORRA-GOBIN, « Planification urbaine et incorporation aux États-Unis, les fondements de la politique d'exclusion ", Annales des la Recherche Urbaine, $\mathrm{n}^{\circ}$ 51, 1992, p. 45.

35. V. RENARD, « La ségrégation par le zonage ", Études Foncières, n 33, 1986, pp. 3-7.

36. Art. cit.

37. Art. cit., p. 90.

38. Op. cit., Chap. IV.

39. Op. cit., p. 54.

\section{RÉSUMÉS}

Le débat autour du Schéma Directeur d'Aménagement et d'Urbanisme de l'Ile-de-France est resté dans le champ strictement politique, par ses enjeux et par son issue. L'objet de ce texte est de poser un regard à la fois critique et comparatif sur les moyens, les structures et les échelles de gestion territoriale, enfin sur les trois grands objectifs contemporains affichés des procédures de planification urbaine et régionale : l'efficacité économique, l'équité territoriale, enfin le respect, voire la stimulation de la démocratie locale.

Urban and regional planning for the Ile-de-France region: lessons and prospect of foreign practices and policies.

In the Ile-de-France region, the debates generated by and revolving around the Schéma Directeur remained strictly confined within the political realm. In this paper, the point is to grasp and identify, through a comparative approach, the means, structures and scales of contemporary urban management procedures and finally the three major objectives/stakes for urban and regional planners to reach, namely: economic efficiency, territorial equity, support to grass roots politics. 
INDEX

Keywords : France, urban and regional planning, Urban management, Comparative approaches Mots-clés : Urbanisme, Ile-de-France, planification urbaine et régionale, Gestion territoriale, Approches comparatives, Schéma Directeur d'Aménagement et d'Urbanisme

\section{AUTEUR}

\section{CATHERINE RHEIN}

Chargée de recherche au CNRS (STRATES), elle poursuit des travaux sur les processus, les formes et les facteurs de la division sociale de l'espace dans l'agglomération centrale de Paris depuis les années 1920. 